\title{
Some Seminormed Difference Sequence Spaces over $n$-Normed Spaces Defined by a Musielak-Orlicz Function of Order $(\alpha, \beta)$
}

\author{
S. A. Mohiuddine $\mathbb{D}^{1},{ }^{1}$ Sunil K. Sharma, ${ }^{2}$ and Dina A. Abuzaid ${ }^{1,3}$ \\ ${ }^{1}$ Operator Theory and Applications Research Group, Department of Mathematics, Faculty of Science, \\ King Abdulaziz University, P.O. Box 80203, Jeddah 21589, Saudi Arabia \\ ${ }^{2}$ Department of Mathematics, Cluster University of Jammu, Jammu 180005, India \\ ${ }^{3}$ Department of Mathematics, Sciences Faculty for Girls, King Abdulaziz University, P.O. Box 4087, Jeddah 21491, Saudi Arabia
}

Correspondence should be addressed to S. A. Mohiuddine; mohiuddine@gmail.com

Received 2 December 2017; Accepted 12 March 2018; Published 22 April 2018

Academic Editor: Maria Alessandra Ragusa

Copyright (C) 2018 S. A. Mohiuddine et al. This is an open access article distributed under the Creative Commons Attribution License, which permits unrestricted use, distribution, and reproduction in any medium, provided the original work is properly cited.

We first define the notion of lacunary statistical convergence of order $(\alpha, \beta)$, and taking this notion into consideration, we introduce some seminormed difference sequence spaces over $n$-normed spaces with the help of Musielak-Orlicz function $\mathscr{M}=\left(M_{k}\right)$ of order $(\alpha, \beta)$. We also examine some topological properties and prove inclusion relations between the resulting sequence spaces.

\section{Introduction and Preliminaries}

Gähler [1] extended the usual notion of normed spaces into 2 -normed spaces, while the notion was again extended to $n$ normed spaces by Misiak [2]. Assume that $X$ is a linear space over the field $\mathbb{K}$ of real or complex numbers of dimension $d \geq$ $n \geq 2, n \in \mathbb{N}$ ( $\mathbb{N}$ denotes the set of natural numbers). A real valued function $\|\cdot, \ldots, \cdot\|$ on $X^{n}$ satisfying the conditions,

(N1) $\left\|x_{1}, x_{2}, \ldots, x_{n}\right\|=0$ if and only if $x_{1}, \ldots, x_{n}$ are linearly dependent in $X$,

(N2) $\left\|x_{1}, x_{2}, \ldots, x_{n}\right\|$ is invariant under permutation,

(N3) $\left\|\alpha x_{1}, x_{2}, \ldots, x_{n}\right\|=|\alpha|\left\|x_{1}, x_{2}, \ldots, x_{n}\right\|$ for any $\alpha \in \mathbb{K}$,

(N4) $\left\|x_{1}+x_{1}^{\prime}, x_{2}, \ldots, x_{n}\right\| \leq\left\|x_{1}, x_{2}, \ldots, x_{n}\right\|+\| x_{1}^{\prime}$, $x_{2}, \ldots, x_{n} \|$,

is called a $n$-norm on $X$, and the pair $(X,\|\cdot, \ldots, \cdot\|)$ is called a $n$-normed space over $\mathbb{K}$.

A sequence $\left(x_{k}\right)_{k \in \mathbb{N}}$ in a $n$-normed space $(X,\|\cdot, \ldots, \cdot\|)$ is said to be

(i) convergent to $\xi \in X$ if $\lim _{k \rightarrow \infty}\left\|x_{k}-\xi, z_{1}, \ldots, z_{n-1}\right\|=$ 0 holds for every $z_{1}, \ldots, z_{n-1} \in X$,

(ii) Cauchy if $\lim _{k, i \rightarrow \infty}\left\|x_{k}-x_{i}, z_{1}, \ldots, z_{n-1}\right\|=0$ holds for every $z_{1}, \ldots, z_{n-1} \in X$.
An $n$-Banach space $(X,\|\cdot, \ldots, \cdot\|)$ is a complete $n$-normed space, where completeness means that, for every $\left(x_{k}\right)$ in $X$ with $\left\|x_{k}-x_{i}, z_{1}, \ldots, z_{n-1}\right\| \rightarrow 0(k, i \rightarrow \infty)$, there exists $\xi \in X$ such that $\left\|x_{k}-\xi, z_{1}, \ldots, z_{n-1}\right\|=0(k \rightarrow \infty)$.

Kizmaz [3] was the first who introduced the idea of difference sequence spaces and studied $Z(\Delta)=\left\{x=\left(x_{k}\right) \in\right.$ $w: \Delta x \in Z\}\left(Z=l_{\infty}, c, c_{0}\right)$, where $\Delta x=x_{k}-x_{k+1}$ for all $k \in \mathbb{N}, w$ is the space of all complex or real sequences, and the standard notations $l_{\infty}, c$, and $c_{0}$ denote bounded, convergent, and null sequences, respectively. Et and Çolak [4] presented a generalization of these difference sequence spaces and introduced the space $Z\left(\Delta^{n}\right)$; in this case, $\Delta^{n} x$ is given by $\Delta^{n} x=\Delta\left(\Delta^{n-1} x\right)=\Delta^{n-1} x_{k}-\Delta^{n-1} x_{k+1}$ for $n \geq 2$. Another generation is given by Tripathy and Esi [5] by considering $\Delta_{m}$ instead as $\Delta$ in Kizmaz spaces and is defined by $\Delta_{m} x=$ $x_{k}-x_{k+m}$. In view of $\Delta^{n}$ and $\Delta_{m}$, Tripathy et al. [6] introduced the difference sequence space as follows:

$$
Z\left(\Delta_{m}^{n}\right)=\left\{x=\left(x_{k}\right) \in w:\left(\Delta_{m}^{n} x_{k}\right) \in Z\right\}
$$

where $\Delta_{m}^{n} x=\left(\Delta_{m}^{n} x_{k}\right)=\left(\Delta_{m}^{n-1} x_{k}-\Delta_{m}^{n-1} x_{k}\right)$ and $\Delta^{0} x_{k}=x_{k}$, which is equivalent to the following binomial representation:

$$
\Delta_{m}^{n} x_{k}=\sum_{v=0}^{n}(-1)^{v}\left(\begin{array}{l}
n \\
v
\end{array}\right) x_{k+m v} .
$$


We remark that if we take $m=1$, then difference sequence space $Z\left(\Delta_{m}^{n}\right)$ is reduced to $Z\left(\Delta^{n}\right)$ and for the choice of $n=$ $m=1$, we obtain the difference sequence space $Z(\Delta)$. For recent work related to various kinds of difference sequence spaces, we refer to [7-13] and references therein.

If $X$ is a linear space and $g: X \rightarrow \mathbb{R}$ such that (i) $x=$ $0 \Rightarrow g(x)=0$, (ii) $g(x+y) \leq g(x)+g(y)$, (iii) $g(-x)=g(x)$, and (iv) if $t_{k} \rightarrow t(k \rightarrow \infty)$ and $x_{k} \rightarrow x(k \rightarrow \infty)$ in the sense that $g\left(x_{k}-x\right) \rightarrow 0(k \rightarrow \infty)$ for scalars $t_{k}, t$ and the vectors $x_{k}, x \in X$, then $t_{k} x_{k} \rightarrow t x(k \rightarrow \infty)$ in the sense that $g\left(t_{k} x_{k}-t x\right) \rightarrow 0(k \rightarrow \infty)$; then $g$ is said to be a paranorm on $X$ and the pair $(X, g)$ is called a paranormed space.

An Orlicz function is a function $M:[0, \infty) \rightarrow[0, \infty)$ which is continuous, nondecreasing, and convex with $M(0)=$ $0, M(x)>0$ as $x>0$ and $M(x) \rightarrow \infty(x \rightarrow \infty)$. Clearly, if $M$ is a convex function and $M(0)=0$, then $M(\lambda x) \leq \lambda M(x)$ for all $\lambda \in(0,1)$. An Orlicz function $M$ is said to satisfy $\Delta_{2}-$ condition for all values of $u$, if there exists a constant $K>$ 0 such that $M(L u) \leq K L M(u)$ for all values of $L>1$ (see Krasnoselskii and Rutitsky [14] and, more recent, Giannetti et al. [15]). Using the idea of Orlicz function, Lindenstrauss and Tzafriri [16] constructed the sequence space

$$
\begin{aligned}
\ell_{M} & =\left\{\left(x_{k}\right) \in w: \sum_{k=1}^{\infty} M\left(\frac{\left|x_{k}\right|}{\rho}\right)<\infty, \text { for some } \rho\right. \\
& >0\},
\end{aligned}
$$

which is called Orlicz sequence space and showed that $\ell_{M}$ is a Banach space with the following norm:

$$
\|x\|=\inf \left\{\rho>0: \sum_{k=1}^{\infty} M\left(\frac{\left|x_{k}\right|}{\rho}\right) \leq 1\right\} .
$$

The space $\ell_{M}$ is closely related to the space $\ell_{p}$ which is an Orlicz sequence space with $M(t)=|t|^{p}$ for $1 \leq p<\infty$.

A sequence $\mathscr{M}=\left(M_{k}\right)$ of Orlicz functions is said to be a Musielak-Orlicz function [17]. A sequence $\mathcal{N}=\left(N_{k}\right)$ is defined by $N_{k}(v)=\sup \left\{|v| u-\left(M_{k}\right): u \geq 0\right\}(k=1,2, \ldots)$ which is said to be complementary function of $\mathscr{M}$. For a given $\mathscr{M}$, the Musielak-Orlicz sequence space $t_{\mathscr{M}}$ and its subspace $h_{\mathscr{M}}$ are defined by

$$
\begin{aligned}
t_{\mathscr{M}} & =\left\{x \in w: I_{\mathscr{M}}(c x)<\infty \text { for some } c>0\right\}, \\
h_{\mathscr{M}} & =\left\{x \in w: I_{\mathscr{M}}(c x)<\infty \forall c>0\right\},
\end{aligned}
$$

where $I_{\mathscr{M}}$ denotes the convex modular and is defined by

$$
I_{\mathscr{M}}(x)=\sum_{k=1}^{\infty}\left(M_{k}\right)\left(x_{k}\right) \quad\left(x=\left(x_{k}\right) \in t_{\mathscr{M}}\right) \text {. }
$$

It is noted that $t_{\mathscr{M}}$ can be considered equipped with the Luxemburg norm or equipped with the Orlicz norm, where Luxemburg and Orlicz norms are given by

$$
\begin{aligned}
\|x\| & =\inf \left\{k>0: I_{\mathscr{M}}\left(\frac{x}{k}\right) \leq 1\right\}, \\
\|x\|^{0} & =\inf \left\{\frac{1}{k}\left(1+I_{\mathscr{M}}(k x)\right): k>0\right\},
\end{aligned}
$$

respectively.
A sequence $x=\left(x_{k}\right) \in l_{\infty}$ is said to be almost convergent if all of its Banach limits coincide. The space $\hat{c}$ of almost convergent sequences was defined by Lorentz [18] (also see [19]) as follows:

$$
\begin{aligned}
\widehat{c} & =\left\{x=\left(x_{k}\right)\right. \\
& \left.\in l_{\infty}: \lim _{n \rightarrow \infty} \frac{1}{n} \sum_{k=1}^{n} x_{k+s} \text { exists, uniformly in } s\right\} .
\end{aligned}
$$

Recall that $x$ is strongly almost convergent [20] to some number $\xi$ if

$$
\lim _{n \rightarrow \infty} \frac{1}{n} \sum_{k=1}^{n}\left|x_{k+s}-\xi\right|=0 \quad \text { uniformly in } s .
$$

The idea of statistical convergence first appeared, under the name of almost convergence, in the first edition of Zygmund [21]. Later, this idea was introduced by Fast [22] and Steinhaus [23], independently, and some of its basic properties were studied by Schoenberg [24], Šalát [25], and Fridy [26]. Some useful results, applications, and various developments on this topic have been presented by many authors; we refer to [27-35]. Çolak [36] extended this notion with the help of $\alpha$-density (for $\alpha=1, \alpha$-density reduced to natural density) and called it statistical convergence of order $\alpha$. In the recent past, Şenül [37] presented an interesting generalization of this notion by taking into account $(\alpha, \beta)$ instead of $\alpha$ and defined statistical convergence of order $(\alpha, \beta)$ as follows.

The sequence $x=\left(x_{k}\right)$ is said to be statistically convergent of order $(\alpha, \beta)$, briefly $S_{\alpha}^{\beta}$-convergence, to $L$ if for each $\epsilon>0$, we have

$$
\lim _{n \rightarrow \infty} \frac{1}{n^{\alpha}}\left|\left\{k \leq n:\left|x_{k}-L\right| \geq \epsilon\right\}\right|^{\beta}=0,
$$

where $|\{k \leq n: k \in E\}|^{\beta}$ denotes the $\beta$ th power of number of elements of $E$ not exceeding $n$, and we write $S_{\alpha}^{\beta}-\lim x_{k}=L$.

\section{Construction of Difference Sequence Spaces}

Before defining some difference sequence spaces, let us first introduce the notion of lacunary statistical convergence of order $(\alpha, \beta)$. Recall that the standard notation $\theta=\left(k_{r}\right)$ denotes the lacunary sequence, where $\left(k_{r}\right)$ is a sequence of positive integers such that $k_{0}=0,0<k_{r}<k_{r+1}$, and $h_{r}:=k_{r}-k_{r-1} \rightarrow \infty(r \rightarrow \infty)$, throughout the article, the intervals determined by the lacunary sequence $\theta$ will be denoted by $I_{r}=\left(k_{r-1}, k_{r}\right]$ and the ratio $q_{r}=k_{r} / k_{r-1} \quad(r \neq 1)$ by $q_{r}$ (see [38]).

Suppose that $E \subset \mathbb{N}$ and $0<\alpha \leq \beta \leq 1$. We define the $\theta(\alpha, \beta)$-density of $E$ by

$$
\delta_{\theta}^{\alpha, \beta}(E)=\lim _{n} \frac{1}{h_{r}^{\alpha}}\left|\left\{k \in\left(k_{r-1}, k_{r}\right]: k \in E\right\}\right|^{\beta},
$$

in case this limit exists, where $h_{r}^{\alpha}$ denotes the $\alpha$ th power of $h_{r}$ and $\left|\left\{k \in I_{r}: k \in E\right\}\right|^{\beta}$ denotes the $\beta$ th power of number of elements of $E$ in $I_{r}$. 
Suppose that $0<\alpha \leq \beta \leq 1$ and let $\theta=\left(k_{r}\right)$ be a lacunary sequence. Then, we say that the sequence $x=\left(x_{k}\right)$ is lacunary statistically convergent of order $(\alpha, \beta)$ (shortly, $S_{\alpha}^{\beta}(\theta)$-convergence) to the number $L$ and we write $S_{\alpha}^{\beta}(\theta)-\lim x_{k}=L$ if for each $\epsilon>0$, the set $A(\epsilon)=\{k \in \mathbb{N}$ : $\left.\left|x_{k}-L\right| \geq \epsilon\right\}$ has $\theta(\alpha, \beta)$-density zero; that is,

$$
\lim _{r \rightarrow \infty} \frac{1}{h_{r}^{\alpha}}\left|\left\{k \in\left(k_{r-1}, k_{r}\right]:\left|x_{k}-L\right| \geq \epsilon\right\}\right|^{\beta}=0 .
$$

Example 1. Consider a sequence $x=\left(x_{k}\right)$ defined by $x_{k}=k$ if $k_{r}-\left[\sqrt{h_{r}^{\alpha}}\right]+1 \leq k \leq k_{r}(r \in \mathbb{N})$ and $x_{k}=0$ otherwise. Also, let $\beta=1$. Then for any $\epsilon>0$,

$$
\begin{aligned}
& \lim _{r \rightarrow \infty} \frac{1}{h_{r}^{\alpha}}\left|\left\{k \in\left(k_{r-1}, k_{r}\right]:\left|x_{k}-0\right| \geq \epsilon\right\}\right| \leq \frac{\left[\sqrt{h_{r}^{\alpha}}\right]}{h_{r}^{\alpha}} \\
& \quad \longrightarrow 0 \quad(r \longrightarrow \infty) .
\end{aligned}
$$

We see that $x=\left(x_{k}\right)$ is $S_{\alpha}^{\beta}(\theta)$-convergent to zero but not convergent.

Remark 2. For $\theta=\left(2^{r}\right)$, the notion of $S_{\alpha}^{\beta}(\theta)$-convergence coincides with $S_{\alpha}^{\beta}$-convergence in [37]. Also, if we take $\theta=$ $\left(2^{r}\right)$ and $\beta=1$, then $S_{\alpha}^{\beta}(\theta)$-convergence reduces to the notion of statistical convergence of order $\alpha$ due to Çolak [36]. Further, the choice of $\theta=\left(2^{r}\right)$ and $\alpha=\beta=1$ in the definition of lacunary statistically convergent of order $(\alpha, \beta)$ gives us the notion of statistical convergence due to Fast [22]. Moreover, if $\alpha=\beta=1$ in the definition of $S_{\alpha}^{\beta}(\theta)$-convergence, then we obtain lacunary statistical convergence introduced by Fridy and Orhan [39].

We denote by $w(n-X)$ the space of all sequences defined over $(X,\|\cdot, \ldots, \cdot\|)$. Let $X$ be a seminormed space, seminormed by $q=\left(q_{k}\right)$, and let $p=\left(p_{k}\right)$ be any bounded sequence of positive real numbers. We are now ready to define the following sequence spaces:

$$
\begin{aligned}
& c^{\theta} {\left[\mathscr{M}, p, \Delta_{n}^{m}, q,\|\cdot \ldots, \cdot\|\right]_{\alpha}^{\beta}=\left\{x=\left(x_{k}\right) \in w(n-X): \lim _{r \rightarrow \infty}\right.} \\
& \cdot \frac{1}{h_{r}^{\alpha}}\left[\sum_{k \in I_{r}}\left[M_{k}\left(q_{k}\left(\left\|\frac{\Delta_{n}^{m} x_{k+s}-L}{\rho}, z_{1}, \ldots, z_{n-1}\right\|\right)\right)\right]^{p_{k}}\right]^{\beta} \\
&\quad=0 \text { uniformly in } s, \text { for some } L, \rho>0\},
\end{aligned}
$$$$
c_{0}^{\theta}\left[\mathscr{M}, p, \Delta_{n}^{m}, q,\|\cdot, \ldots, \cdot\|\right]_{\alpha}^{\beta}=\left\{x=\left(x_{k}\right) \in w(n-X): \lim _{r \rightarrow \infty}\right.
$$$$
\cdot \frac{1}{h_{r}^{\alpha}}\left[\sum_{k \in I_{r}}\left[M_{k}\left(q_{k}\left(\left\|\frac{\Delta_{n}^{m} x_{k+s}}{\rho}, z_{1}, \ldots, z_{n-1}\right\|\right)\right)\right]^{p_{k}}\right]^{\beta}
$$$$
=0 \text { uniformly in } s \text {, for some } \rho>0\} \text {, }
$$

$$
\begin{aligned}
& c_{\infty}^{\theta}\left[\mathscr{M}, p, \Delta_{n}^{m}, q,\|\cdot, \ldots, \cdot\|\right]_{\alpha}^{\beta}=\left\{x=\left(x_{k}\right) \in w(n-X): \sup _{r, s}\right. \\
& \cdot \frac{1}{h_{r}^{\alpha}}\left[\sum_{k=1}^{n}\left[M_{k}\left(q_{k}\left(\left\|\frac{\Delta_{n}^{m} x_{k+s}}{\rho}, z_{1}, \ldots, z_{n-1}\right\|\right)\right)\right]^{p_{k}}\right]^{\beta} \\
& <\infty \text { uniformly in } s, \text { for some } \rho>0\} .
\end{aligned}
$$

Note that if we consider $\mathscr{M}(x)=x$; then the above difference sequence spaces reduce to the following spaces:

$$
\begin{aligned}
& c^{\theta}\left[p, \Delta_{n}^{m}, q,\|\cdot, \ldots, \cdot\|\right]_{\alpha}^{\beta}=\left\{x=\left(x_{k}\right) \in w(n-X): \lim _{r \rightarrow \infty}\right. \\
& \cdot \frac{1}{h_{r}^{\alpha}}\left[\sum_{k \in I_{r}}\left(q_{k}\left(\left\|\frac{\Delta_{n}^{m} x_{k+s}-L}{\rho}, z_{1}, \ldots, z_{n-1}\right\|\right)\right)^{p_{k}}\right]^{\beta} \\
& =0 \text { uniformly in } s, \text { for some } L, \rho>0\}, \\
& c_{0}^{\theta}\left[p, \Delta_{n}^{m}, q,\|\cdot, \ldots, \cdot\|\right]_{\alpha}^{\beta}=\left\{x=\left(x_{k}\right) \in w(n-X): \lim _{r \rightarrow \infty}\right. \\
& \cdot \frac{1}{h_{r}^{\alpha}}\left[\sum_{k \in I_{r}}\left(q_{k}\left(\left\|\frac{\Delta_{n}^{m} x_{k+s}}{\rho}, z_{1}, \ldots, z_{n-1}\right\|\right)\right)^{p_{k}}\right]^{\beta} \\
& \quad \cdot \frac{1}{h_{r}^{\alpha}}\left[\sum_{k=1}^{n}\left(q_{k}\left(\left\|\frac{\Delta_{n}^{m} x_{k+s}}{\rho}, z_{1}, \ldots, z_{n-1}\right\|\right)\right)^{p_{k}}\right]^{\beta} \\
& c_{\infty}^{\theta}\left[p, \Delta_{n}^{m}, q,\|\cdot, \ldots,\|\right]_{\alpha}^{\beta}=\left\{x=\left(x_{k}\right) \in w(n-X): \sup _{r, s}\right. \\
& \quad 0 \text { uniformly in } s, \text { for some } \rho>0\},
\end{aligned}
$$

It is to further notice that if we take $p_{k}=1$ for all $k \epsilon$ $\mathbb{N}$, then the spaces $c^{\theta}\left[\mathscr{M}, p, \Delta_{n}^{m}, q,\|\cdot, \ldots, \cdot\|\right]_{\alpha}^{\beta}, c_{0}^{\theta}\left[\mathscr{M}, p, \Delta_{n}^{m}\right.$, $q,\|\cdot, \ldots, \cdot\|]_{\alpha}^{\beta}$, and $c_{\infty}^{\theta}\left[\mathscr{M}, p, \Delta_{n}^{m}, q,\|\cdot, \ldots, \cdot\|\right]_{\alpha}^{\beta}$ reduce to the following spaces:

$$
\begin{aligned}
& c^{\theta}\left[\mathscr{M}, \Delta_{n}^{m}, q,\|\cdot, \ldots, \cdot\|\right]_{\alpha}^{\beta}=\left\{x=\left(x_{k}\right) \in w(n-X): \lim _{r \rightarrow \infty}\right. \\
& \cdot \frac{1}{h_{r}^{\alpha}}\left[\sum_{k \in I_{r}}\left[M_{k}\left(q_{k}\left(\left\|\frac{\Delta_{n}^{m} x_{k+s}-L}{\rho}, z_{1}, \ldots, z_{n-1}\right\|\right)\right)\right]\right]^{\beta} \\
& =0 \text {, uniformly in } s, \text { for some } L, \rho>0\},
\end{aligned}
$$




$$
\begin{aligned}
& c_{0}^{\theta}\left[\mathscr{M}, \Delta_{n}^{m}, q,\|\cdot, \ldots, \cdot\|\right]_{\alpha}^{\beta}=\left\{x=\left(x_{k}\right) \in w(n-X): \lim _{r \rightarrow \infty}\right. \\
& \cdot \frac{1}{h_{r}^{\alpha}}\left[\sum_{k \in I_{r}}\left[M_{k}\left(q_{k}\left(\left\|\frac{\Delta_{n}^{m} x_{k+s}}{\rho}, z_{1}, \ldots, z_{n-1}\right\|\right)\right)\right]\right]^{\beta} \\
& =0, \text { uniformly in } s, \text { for some } \rho>0\}, \\
& c_{\infty}^{\theta}\left[\mathscr{M}, \Delta_{n}^{m}, q,\|\cdot, \ldots, \cdot\|\right]_{\alpha}^{\beta}=\left\{x=\left(x_{k}\right) \in w(n-X): \sup _{r, s}\right. \\
& \cdot \frac{1}{h_{r}^{\alpha}}\left[\sum_{k=1}^{n}\left[M_{k}\left(q_{k}\left(\left\|\frac{\Delta_{n}^{m} x_{k+s}}{\rho}, z_{1}, \ldots, z_{n-1}\right\|\right)\right)\right]\right]^{\beta} \\
& \quad<\infty, \text { uniformly in } s, \text { for some } \rho>0\} .
\end{aligned}
$$

Remark 3. If $\alpha=\beta=1, n=1$, and $q_{k}(x)=x \forall k$ in our difference sequence spaces defined above, then these spaces reduce to the difference sequence spaces defined by Raj et al. [40]. Moreover, if we take $\alpha=\beta=1$ in our difference sequence spaces and take $u_{k}=1$ for all $k$ in the difference sequence spaces defined by Raj and Sharma [41], then we observe that difference sequence spaces of both the manuscripts coincide.

The following inequality will be used to prove some of our results in the next section. If $0 \leq p_{k} \leq \sup p_{k}=H, D=$ $\max \left(1,2^{H-1}\right)$, then

$$
\left|a_{k}+b_{k}\right|^{p_{k}} \leq D\left\{\left|a_{k}\right|^{p_{k}}+\left|b_{k}\right|^{p_{k}}\right\}
$$

for all $k$ and $a_{k}, b_{k} \in \mathbb{C}$. Also $|a|^{p_{k}} \leq \max \left(1,|a|^{H}\right)$ for all $a \in \mathbb{C}$.

\section{Main Results}

In this section, we are going to study some topological properties and to obtain some inclusion relations between the difference sequence spaces defined in the previous section.

Theorem 4. Let $\mathscr{M}=\left(M_{k}\right)$ be a Musielak-Orlicz function and let $p=\left(p_{k}\right)$ be a bounded sequence of positive real numbers. Then the spaces $c^{\theta}\left[\mathscr{M}, p, \Delta_{n}^{m}, q,\|\cdot, \ldots, \cdot\|\right]_{\alpha}^{\beta}$, $c_{0}^{\theta}\left[\mathscr{M}, p, \Delta_{n}^{m}, q,\|\cdot, \ldots, \cdot\|\right]_{\alpha}^{\beta}$ and $c_{\infty}^{\theta}\left[\mathscr{M}, p, \Delta_{n}^{m}, q,\|\cdot \ldots, \cdot\|\right]_{\alpha}^{\beta}$ are linear over the field of complex numbers $\mathbb{C}$.

Proof. Let $x=\left(x_{k}\right), y=\left(y_{k}\right) \in c_{0}^{\theta}\left[\mathscr{M}, p, \Delta_{n}^{m}, q,\|\cdot, \ldots, \cdot\|\right]_{\alpha}^{\beta}$ and $\alpha, \beta \in \mathbb{C}$. Then there exist positive numbers $\rho_{1}$ and $\rho_{2}$ such that

$$
\begin{aligned}
& \lim _{r \rightarrow \infty} \frac{1}{h_{r}^{\alpha}}\left[\sum_{k \in I_{r}}\left[M_{k}\left(q_{k}\left(\left\|\frac{\Delta_{n}^{m} x_{k+s}}{\rho_{1}}, z_{1}, \ldots, z_{n-1}\right\|\right)\right)\right]^{p_{k}}\right]^{\beta} \\
& =0 \text { uniformly in } s, \\
& \lim _{r \rightarrow \infty} \frac{1}{h_{r}^{\alpha}}\left[\sum_{k \in I_{r}}\left[M_{k}\left(q_{k}\left(\left\|\frac{\Delta_{n}^{m} x_{k+s}}{\rho_{2}}, z_{1}, \ldots, z_{n-1}\right\|\right)\right)\right]^{p_{k}}\right]^{\beta}
\end{aligned}
$$

$$
=0 \quad \text { uniformly in } s \text {. }
$$

Let $\rho_{3}=\max \left(2|\alpha| \rho_{1}, 2|\beta| \rho_{2}\right)$. Since $\mathscr{M}=\left(M_{k}\right)$ is nondecreasing convex function, by (17), we obtain

$$
\begin{aligned}
\frac{1}{h_{r}^{\alpha}}\left[\sum_{k \in I_{r}}\left[M_{k}\left(q_{k}\left(\left\|\frac{\Delta_{n}^{m}\left(\alpha x_{k+s}+\beta y_{k+s}\right)}{\rho_{3}}, z_{1}, \ldots, z_{n-1}\right\|\right)\right)\right]^{p_{k}}\right]^{\beta} \\
\leq D \frac{1}{h_{r}^{\alpha}}\left[\sum_{k \in I_{r}} \frac{1}{2^{p_{k}}}\left[M_{k}\left(q_{k}\left(\left\|\frac{\Delta_{n}^{m}\left(x_{k+s}\right)}{\rho_{1}}, z_{1}, \ldots, z_{n-1}\right\|\right)\right)\right]^{p_{k}}\right]^{\beta} \\
+D \frac{1}{h_{r}^{\alpha}}\left[\sum_{k \in I_{r}} \frac{1}{2_{p_{k}}}\left[M_{k}\left(q_{k}\left(\left\|\frac{\Delta_{n}^{m}\left(y_{k+s}\right)}{\rho_{2}}, z_{1}, \ldots, z_{n-1}\right\|\right)\right)\right]^{p_{k}}\right]^{\beta} \\
\left.\leq D \frac{1}{h_{r}^{\alpha}}\left[\sum_{k \in I_{r}}\left[M_{k}\left(q_{k}\left(\left\|\frac{\Delta_{n}^{m}\left(x_{k+s}\right)}{\rho_{1}}, z_{1}, \ldots, z_{n-1}\right\|\right)\right)\right]^{p_{k}}\right]^{\beta}\right]^{\beta} \\
\quad+D \frac{1}{h_{r}^{\alpha}}\left[\sum_{k \in I_{r}}\left[M_{k}\left(q_{k}\left(\left\|\frac{\Delta_{n}^{m}\left(y_{k+s}\right)}{\rho_{2}}, z_{1}, \ldots, z_{n-1}\right\|\right)\right)\right]^{p_{k}}\right]^{\beta} \longrightarrow 0 \text { as } r \longrightarrow \text { uniformly in } s .
\end{aligned}
$$


Thus, we have $(\alpha x+\beta y) \in c_{0}^{\theta}\left[\mathscr{M}, p, \Delta_{n}^{m}, q,\|\cdot, \ldots, \cdot\|\right]_{\alpha}^{\beta}$. Hence, $c_{0}^{\theta}\left[\mathscr{M}, p, \Delta_{n}^{m}, q,\|\cdot \ldots, \cdot\|\right]_{\alpha}^{\beta}$ is a linear space. In a similar way, one can prove that $c^{\theta}\left[\mathscr{M}, p, \Delta_{n}^{m}, q,\|\cdot, \ldots, \cdot\|\right]_{\alpha}^{\beta}$ and $c_{\infty}^{\theta}\left[\mathscr{M}, p, \Delta_{n}^{m}, q,\|\cdot, \ldots, \cdot\|\right]_{\alpha}^{\beta}$ are linear spaces.

Theorem 5. For any Musielak-Orlicz function $\mathscr{M}=\left(M_{k}\right)$ and a bounded sequence of positive real numbers $p=\left(p_{k}\right)$, the space $c_{0}^{\theta}\left[\mathscr{M}, p, \Delta_{n}^{m}, q,\|\cdot, \ldots, \cdot\|\right]_{\alpha}^{\beta}$ is a topological linear space paranormed by

$$
\begin{aligned}
& g(x)=\inf \left\{\rho^{p_{r} / K}:\right. \\
& \left(\frac{1}{h_{r}^{\alpha}}\left[\sum_{k \in I_{r}}\left[M_{k}\left(q_{k}\left(\left\|\frac{\Delta_{n}^{m} x_{k+s}}{\rho}, z_{1}, \ldots, z_{n-1}\right\|\right)\right)\right]^{p_{k}}\right]^{\beta}\right)^{1 / K} \\
& \leq 1, r, s \in \mathbb{N}\},
\end{aligned}
$$

where $K=\max \left(1, \sup _{k} p_{k}<\infty\right)$.

Proof. Clearly $g(x) \geq 0$ for $x=\left(x_{k}\right) \in c_{0}^{\theta}[\mathscr{M}, p$, $\left.\Delta_{n}^{m}, q,\|\cdot, \ldots, \cdot\|\right]_{\alpha}^{\beta}$. Since $M_{k}(0)=0$, we obtain $g(0)=0$. Again, if $g(x)=0$, then we have

$$
\begin{aligned}
& \inf \left\{\rho^{p_{r} / K}:\right. \\
& \left(\frac{1}{h_{r}^{\alpha}}\left[\sum_{k \in I_{r}}\left[M_{k}\left(q_{k}\left(\left\|\frac{\Delta_{n}^{m} x_{k+s}}{\rho}, z_{1}, \ldots, z_{n-1}\right\|\right)\right)\right]^{p_{k}}\right]^{\beta}\right)^{1 / K} \\
& \leq 1, r, s \in \mathbb{N}\}=0 .
\end{aligned}
$$

This implies that, for a given $\epsilon>0$, there exists some $\rho_{\epsilon}(0<$ $\left.\rho_{\epsilon}<\epsilon\right)$ such that

$$
\left(\frac{1}{h_{r}^{\alpha}}\left[\sum_{k \in I_{r}}\left[M_{k}\left(q_{k}\left(\left\|\frac{\Delta_{n}^{m} x_{k+s}}{\rho_{\epsilon}}, z_{1}, \ldots, z_{n-1}\right\|\right)\right)\right]^{p_{k}}\right]^{\beta}\right)^{1 / K}
$$

$$
\leq 1 \text {. }
$$

Thus,

$$
\begin{aligned}
& \left(\frac{1}{h_{r}^{\alpha}}\left[\sum_{k \in I_{r}}\left[M_{k}\left(q_{k}\left(\left\|\frac{\Delta_{n}^{m} x_{k+s}}{\epsilon}, z_{1}, \ldots, z_{n-1}\right\|\right)\right)\right]^{p_{k}}\right]^{\beta}\right)^{1 / K} \\
& \leq\left(\frac{1}{h_{r}^{\alpha}}\left[\sum_{k \in I_{r}}\left[M_{k}\left(q_{k}\left(\left\|\frac{\Delta_{n}^{m} x_{k+s}}{\rho_{\epsilon}}, z_{1}, \ldots, z_{n-1}\right\|\right)\right)\right]^{p_{k}}\right]^{\beta}\right)^{1 / K}
\end{aligned}
$$

$\leq 1$,

for each $r$ and $s$. Assume that $x_{k} \neq 0$ for each $k \in \mathbb{N}$. This implies that $\Delta_{n}^{m} x_{k+s} \neq 0$ for each $k, s \in \mathbb{N}$. Let $\epsilon \rightarrow 0$; then $q_{k}\left(\left\|\Delta_{n}^{m} x_{k+s} / \epsilon, z_{1}, \ldots, z_{n-1}\right\|\right) \rightarrow \infty$. It follows that

$$
\left(\frac{1}{h_{r}^{\alpha}}\left[\sum_{k \in I_{r}}\left[M_{k}\left(q_{k}\left(\left\|\frac{\Delta_{n}^{m} x_{k+s}}{\epsilon}, z_{1}, \ldots, z_{n-1}\right\|\right)\right)\right]^{p_{k}}\right]^{\beta}\right)^{1 / K}
$$

$\longrightarrow \infty$,

which is a contradiction. Consequently, we have $\Delta_{n}^{m} x_{k+s}=0$ for each $k, s$ and so $x_{k}=0$ for each $k \in \mathbb{N}$. Suppose that $\rho_{1}>0$ and $\rho_{2}>0$ be such that

$$
\begin{aligned}
& \left(\frac{1}{h_{r}^{\alpha}}\left[\sum_{k \in I_{r}}\left[M_{k}\left(q_{k}\left(\left\|\frac{\Delta_{n}^{m} x_{k+s}}{\rho_{1}}, z_{1}, \ldots, z_{n-1}\right\|\right)\right)\right]^{p_{k}}\right]^{\beta}\right)^{1 / K} \\
& \quad \leq 1, \\
& \left(\frac{1}{h_{r}^{\alpha}}\left[\sum_{k \in I_{r}}\left[M_{k}\left(q_{k}\left(\left\|\frac{\Delta_{n}^{m} x_{k+s}}{\rho_{2}}, z_{1}, \ldots, z_{n-1}\right\|\right)\right)\right]^{p_{k}}\right]^{\beta}\right)^{1 / K} \\
& \quad \leq 1,
\end{aligned}
$$

for each $r$ and $s$. Assume that $\rho=\rho_{1}+\rho_{2}$. Then, by Minkowski's inequality, we have

$$
\begin{aligned}
& \left(\frac{1}{h_{r}^{\alpha}}\left[\sum_{k \in I_{r}}\left[M_{k}\left(q_{k}\left(\left\|\frac{\Delta_{n}^{m}\left(x_{k+s}+y_{k+s}\right)}{\rho}, z_{1}, \ldots, z_{n-1}\right\|\right)\right)\right]^{p_{k}}\right]^{\beta}\right)^{1 / K} \\
& \leq\left(\left[\sum_{k \in I_{r}}\left[\frac{\rho_{1}}{\rho_{1}+\rho_{2}} M_{k}\left(q_{k}\left(\left\|\frac{\Delta_{n}^{m}\left(x_{k+s}\right)}{\rho_{1}}, z_{1}, \ldots, z_{n-1}\right\|\right)\right)+\frac{\rho_{2}}{\rho_{1}+\rho_{2}} M_{k}\left(q_{k}\left(\left\|\frac{\Delta_{n}^{m}\left(y_{k+s}\right)}{\rho_{2}}, z_{1}, \ldots, z_{n-1}\right\|\right)\right)\right]^{p_{k}}\right]^{\beta}\right)^{1 / K} \\
& \left.\left.\leq\left(\frac{\rho_{1}}{\rho_{1}+\rho_{2}}\right)\left(\frac{1}{h_{r}^{\alpha}}\left[\sum_{k \in I_{r}}\left[M_{k}\left(q_{k}\left(\left\|\frac{\Delta_{n}^{m}\left(x_{k+s}\right)}{\rho_{1}}, z_{1}, \ldots, z_{n-1}\right\|\right)\right)\right]^{p_{k}}\right]^{\beta}\right)^{1 / K}\right)^{\rho_{2}}\right)\left(\frac{1}{h_{r}^{\alpha}}\left[\sum_{k \in I_{r}}\left[M_{k}\left(q_{k}\left(\left\|\frac{\Delta_{n}^{m}\left(y_{k+s}\right)}{\rho_{2}}, z_{1}, \ldots, z_{n-1}\right\|\right)\right)\right]^{p_{k}}\right]^{\beta}\right)^{1 / K} \leq 1 .
\end{aligned}
$$


Since $\rho$ 's are nonnegative, one obtains

$$
\begin{aligned}
g(x+y)= & \inf \left\{\rho^{p_{r} / K}:\left(\frac{1}{h_{r}^{\alpha}}\left[\sum_{k \in I_{r}}\left[M_{k}\left(q_{k}\left(\left\|\frac{\Delta_{n}^{m}\left(x_{k+s}+y_{k+s}\right)}{\rho}, z_{1}, \ldots, z_{n-1}\right\|\right)\right)\right]^{p_{k}}\right]^{\beta}\right)^{1 / K} \leq 1, r, s \in \mathbb{N}\right\} \\
\leq & \inf \left\{\rho_{1}^{p_{r} / K}:\left(\frac{1}{h_{r}^{\alpha}}\left[\sum_{k \in I_{r}}\left[M_{k}\left(q_{k}\left(\left\|\frac{\Delta_{n}^{m}\left(x_{k+s}\right)}{\rho_{1}}, z_{1}, \ldots, z_{n-1}\right\|\right)\right)\right]^{p_{k}}\right]^{\beta} \leq 1, r, s \in \mathbb{N}\right\}\right. \\
& +\inf \left\{\rho_{2}^{p_{r} / K}:\left(\frac{1}{h_{r}^{\alpha}}\left[\sum_{k \in I_{r}}\left[M_{k}\left(q_{k}\left(\left\|\frac{\Delta^{m}\left(y_{k+s}\right)}{\rho_{2}}, z_{1}, \ldots, z_{n-1}\right\|\right)\right)\right]^{p_{k}}\right]^{\beta}\right)^{1 / K} \leq 1, r, s \in \mathbb{N}\right\} .
\end{aligned}
$$

Consequently, $g(x+y) \leq g(x)+g(y)$. It is only left to show that the scalar multiplication is continuous. Let us consider a complex number $\lambda$. Then, by definition, we obtain

$$
\begin{aligned}
& g(\lambda x)=\inf \left\{\rho^{p_{r} / K}:\right. \\
& \left(\frac{1}{h_{r}^{\alpha}}\left[\sum_{k \in I_{r}}\left[M_{k}\left(q_{k}\left(\left\|\frac{\Delta_{n}^{m} \lambda x_{k+s}}{\rho}, z_{1}, \ldots, z_{n-1}\right\|\right)\right)\right]^{p_{k}}\right]^{\beta}\right)^{1 / K} \\
& \leq 1, r, s \in \mathbb{N}\} .
\end{aligned}
$$

Then

$$
\begin{aligned}
& g(\lambda x)=\inf \left\{(|\lambda| t)^{p_{r} / K}:\right. \\
& \left(\frac{1}{h_{r}^{\alpha}}\left[\sum_{k \in I_{r}}\left[M_{k}\left(q_{k}\left(\left\|\frac{\Delta^{m} x_{k+s}}{t}, z_{1}, \ldots, z_{n-1}\right\|\right)\right)\right]^{p_{k}}\right]^{\beta}\right)^{1 / K} \\
& \leq 1, r, s \in \mathbb{N}\},
\end{aligned}
$$

where $t=\rho /|\lambda|$. Since $|\lambda|^{p_{r}} \leq \max \left(1,|\lambda|^{\text {sup } p_{r}}\right)$, we have

$$
\begin{aligned}
& g(\lambda x) \leq \max \left(1,|\lambda|^{\text {supp } p_{r}}\right) \inf \left\{t^{p_{r} / K}:\right. \\
& \left(\frac{1}{h_{r}^{\alpha}}\left[\sum_{k \in I_{r}}\left[M_{k}\left(q_{k}\left(\left\|\frac{\Delta_{n}^{m} x_{k+s}}{t}, z_{1}, \ldots, z_{n-1}\right\|\right)\right)\right]^{p_{k}}\right]^{\beta}\right)^{1 / K} \\
& \leq 1, r, s \in \mathbb{N}\} .
\end{aligned}
$$

So, the fact that scalar multiplication is continuous follows from the above inequality.

Theorem 6. Let $\mathscr{M}=\left(M_{k}\right)$ be a Musielak-Orlicz function. If $\sup _{k}\left[M_{k}(x)\right]^{p_{k}}<\infty$ for all fixed $x>0$, then $c_{0}^{\theta}\left[\mathscr{M}, p, \Delta_{n}^{m}, q,\|\cdot, \ldots, \cdot\|\right]_{\alpha}^{\beta} \subset c_{\infty}^{\theta}\left[\mathscr{M}, p, \Delta_{n}^{m}, q,\|\cdot, \ldots, \cdot\|\right]_{\alpha}^{\beta}$.

Proof. Let $x=\left(x_{k}\right) \in c_{0}^{\theta}\left[\mathscr{M}, p, \Delta_{n}^{m}, q,\|\cdot, \ldots, \cdot\|\right]_{\alpha}^{\beta}$. There exists some positive $\rho_{1}$ such that

$$
\begin{aligned}
& \lim _{r \rightarrow \infty} \frac{1}{h_{r}^{\alpha}}\left[\sum_{k \in I_{r}}\left[M_{k}\left(q_{k}\left(\left\|\frac{\Delta_{n}^{m} x_{k+s}}{\rho_{1}}, z_{1}, \ldots, z_{n-1}\right\|\right)\right)\right]^{p_{k}}\right]^{\beta} \\
& =0 \text { uniformly in } s .
\end{aligned}
$$

Define $\rho=2 \rho_{1}$. Since $\mathscr{M}=\left(M_{k}\right)$ is nondecreasing and convex, by using (17), we obtain

$$
\begin{aligned}
\sup _{r, s} & \frac{1}{h_{r}^{\alpha}}\left[\sum_{k \in I_{r}}\left[M_{k}\left(q_{k}\left(\left\|\frac{\Delta_{n}^{m} x_{k+s}}{\rho}, z_{1}, \ldots, z_{n-1}\right\|\right)\right)\right]^{p_{k}}\right]^{\beta} \\
\leq & D \sup _{r, s} \frac{1}{h_{r}^{\alpha}}\left[\sum _ { k \in I _ { r } } \left[\frac{1}{2^{p_{k}}}\right.\right. \\
\cdot & \left.\left.M_{k}\left(q_{k}\left(\left\|\frac{\Delta_{n}^{m} x_{k+s}-L}{\rho_{1}}, z_{1}, \ldots, z_{n-1}\right\|\right)\right)\right]^{p_{k}}\right]^{\beta}+D \sup _{r, s}^{\beta} \\
\cdot & \frac{1}{h_{r}^{\alpha}}\left[\sum_{k \in I_{r}}\left[\frac{1}{2^{p_{k}}} M_{k}\left(q_{k}\left(\left\|\frac{L}{\rho_{1}}, z_{1}, \ldots, z_{n-1}\right\|\right)\right)\right]^{p_{k}}\right]^{\beta} \\
\leq & D \sup _{r, s} \\
\cdot & \frac{1}{h_{r}^{\alpha}}\left[\sum_{k \in I_{r}}\left[M_{k}\left(q_{k}\left(\left\|\frac{\Delta_{n}^{m} x_{k+s}-L}{\rho_{1}}, z_{1}, \ldots, z_{n-1}\right\|\right)\right)^{p_{k}}\right]^{\beta}\right. \\
+ & D \sup _{r, s} \frac{1}{h_{r}^{\alpha}}\left[\sum_{k \in I_{r}}\left[M_{k}\left(q_{k}\left(\left\|\frac{L}{\rho_{1}}, z_{1}, \ldots, z_{n-1}\right\|\right)\right)\right]^{p_{k}}\right]^{\beta},
\end{aligned}
$$


which gives

$$
\begin{aligned}
& \sup _{r, s} \frac{1}{h_{r}^{\alpha}}\left[\sum_{k \in I_{r}}\left[M_{k}\left(q_{k}\left(\left\|\frac{\Delta_{n}^{m} x_{k+s}}{\rho}, z_{1}, \ldots, z_{n-1}\right\|\right)\right)\right]^{p_{k}}\right]^{\beta} \\
& \quad<\infty .
\end{aligned}
$$

Hence, $x=\left(x_{k}\right) \in c_{\infty}^{\theta}\left[\mathscr{M}, p, \Delta_{n}^{m}, q,\|\cdot, \ldots, \cdot\|\right]_{\alpha}^{\beta}$.

Theorem 7. Let $0<\inf p_{k}=h \leq p_{k} \leq \sup p_{k}=H<\infty$ and let $\mathscr{M}=\left(M_{k}\right)$ and $\mathscr{M}^{\prime}=\left(M_{k}^{\prime}\right)$ be two Musielak-Orlicz functions satisfying $\Delta_{2}$-condition. Then

(i) $c_{0}^{\theta}\left[\mathscr{M}^{\prime}, p, \Delta_{n}^{m}, q,\|\cdot, \ldots, \cdot\|\right]_{\alpha}^{\beta} \subset c_{0}^{\theta}\left[\mathscr{M} \circ \mathscr{M}^{\prime}, p, \Delta_{n}^{m}, q\right.$, $\|\cdot, \ldots, \cdot\|]_{\alpha}^{\beta}$

(ii) $c^{\theta}\left[\mathscr{M}^{\prime}, p, \Delta_{n}^{m}, q,\|\cdot, \ldots, \cdot\|\right]_{\alpha}^{\beta} \subset c^{\theta}\left[\mathscr{M} \circ \mathscr{M}^{\prime}, p, \Delta_{n}^{m}, q\right.$, $\|\cdot, \ldots, \cdot\|]_{\alpha}^{\beta}$

(iii) $c_{\infty}^{\theta}\left[\mathscr{M}^{\prime}, p, \Delta_{n}^{m}, q,\|\cdot, \ldots, \cdot\|\right]_{\alpha}^{\beta} \subset c_{\infty}^{\theta}\left[\mathscr{M} \circ \mathscr{M}^{\prime}, p, \Delta_{n}^{m}, q\right.$, $\|\cdot, \ldots, \cdot\|]_{\alpha}^{\beta}$.

Proof. Let $x=\left(x_{k}\right) \in c^{\theta}\left[\mathscr{M}, p, \Delta_{n}^{m}, q,\|\cdot, \ldots, \cdot\|\right]_{\alpha}^{\beta}$. Then, we have

$$
\begin{aligned}
& \lim _{r \rightarrow \infty} \frac{1}{h_{r}^{\alpha}}\left[\sum_{k \in I_{r}}\left[M_{k}^{\prime}\left(q_{k}\left(\left\|\frac{\Delta_{n}^{m} x_{k+s}-L}{\rho}, z_{1}, \ldots, z_{n-1}\right\|\right)\right)\right]^{p_{k}}\right]^{\beta} \\
& \quad=0
\end{aligned}
$$

uniformly in $s$ and for some $L \in \mathbb{C}$. Let $\epsilon>0$ and choose $\delta$ with $0<\delta<1$ such that $M_{k}(t)<\epsilon$ for $0 \leq t \leq \delta$. Let

$$
y_{k, s}=M_{k}^{\prime}\left(q_{k}\left(\left\|\frac{\Delta_{n}^{m} x_{k+s}-L}{\rho}, z_{1}, \ldots, z_{n-1}\right\|\right)\right)
$$

$\forall k, s \in \mathbb{N}$.

We can write

$$
\begin{aligned}
\frac{1}{h_{r}^{\alpha}}\left[\sum_{k \in I_{r}}\left[M_{k}\left(y_{k, s}\right)\right]^{p_{k}}\right]^{\beta} \\
=\frac{1}{h_{r}^{\alpha}}\left[\sum_{k \in I_{r}, y_{k, s} \leq \delta}\left[M_{k}\left(y_{k, s}\right)\right]^{p_{k}}\right]^{\beta} \\
+\frac{1}{h_{r}^{\alpha}}\left[\sum_{k \in I_{r}, y_{k, s}>\delta}\left[M_{k}\left(y_{k, s}\right)\right]^{p_{k}}\right]^{\beta} .
\end{aligned}
$$

Since $\mathscr{M}=\left(M_{k}\right)$ satisfies $\Delta_{2}$-condition, we have

$$
\begin{aligned}
& \frac{1}{h_{r}^{\alpha}}\left[\sum_{k \in I_{r}, y_{k, s} \leq \delta}\left[M_{k}\left(y_{k, s}\right)\right]^{p_{k}}\right]^{\beta} \\
& \quad \leq\left[M_{k}(1)\right]^{H} \frac{1}{h_{r}^{\alpha}}\left[\sum_{k \in I_{r}, y_{k, s} \leq \delta}\left[M_{k}\left(y_{k, s}\right)\right]^{p_{k}}\right]^{\beta} .
\end{aligned}
$$

For $y_{k, s}>\delta$, we obtain

$$
y_{k, s}<\frac{y_{k, s}}{\delta}<1+\frac{y_{k, s}}{\delta} .
$$

It follows from the fact that $\mathscr{M}=\left(M_{k}\right)$ is nondecreasing and convex that

$$
\begin{aligned}
M_{k}\left(y_{k, s}\right) & <M_{k}\left(1+\frac{y_{k, s}}{\delta}\right) \\
& <\frac{1}{2} M_{k}(2)+\frac{1}{2} M_{k}\left(\frac{2 y_{k, s}}{\delta}\right) .
\end{aligned}
$$

Since $\left(M_{k}\right)$ satisfies $\Delta_{2}$-condition, we can write

$$
\begin{aligned}
M_{k}\left(y_{k, s}\right) & <\frac{1}{2} T \frac{y_{k, s}}{\delta} M_{k}(2)+\frac{1}{2} T \frac{y_{k, s}}{\delta} M_{k}(2) \\
& =T \frac{y_{k, s}}{\delta} M_{k}(2) .
\end{aligned}
$$

Hence,

$$
\begin{gathered}
\frac{1}{h_{r}^{\alpha}}\left[\sum_{k \in I_{r}, y_{k, s}>\delta}\left[M_{k}\left(y_{k, s}\right)\right]^{p_{k}}\right]^{\beta} \\
\leq \max \left(1,\left(\frac{T M_{k}(2)}{\delta}\right)^{H}\right) \\
\cdot \frac{1}{h_{r}^{\alpha}}\left[\sum_{k \in I_{r}, y_{k, s}>\delta}\left[\left(y_{k, s}\right)\right]^{p_{k}}\right]^{\beta} .
\end{gathered}
$$

Equation (37) together with (41) gives

$$
x=\left(x_{k}\right) \in c_{0}^{\theta}\left[\mathscr{M} \circ \mathscr{M}^{\prime}, p, \Delta_{n}^{m}, q,\|\cdot, \ldots, \cdot\|\right]_{\alpha}^{\beta} .
$$

This completes the proof of (i). In a similar way, using $\Delta_{2}$ condition for $\left(M_{k}\right)$, we can prove that

$$
\begin{aligned}
& c^{\theta}\left[\mathscr{M}^{\prime}, p, \Delta_{n}^{m}, q,\|\cdot, \ldots, \cdot\|\right]_{\alpha}^{\beta} \\
& \quad \subset c^{\theta}\left[\mathscr{M}^{\prime} \circ \mathscr{M}^{\prime}, p, \Delta_{n}^{m}, q,\|\cdot, \ldots, \cdot\|\right]_{\alpha}^{\beta}, \\
& c_{\infty}^{\theta}\left[\mathscr{M}^{\prime}, p, \Delta_{n}^{m}, q,\|\cdot, \ldots, \cdot\|\right]_{\alpha}^{\beta} \\
& \quad \subset c_{\infty}^{\theta}\left[\mathscr{M}_{0} \circ \mathscr{M}^{\prime}, p, \Delta_{n}^{m}, q,\|\cdot, \ldots, \cdot\|\right]_{\alpha}^{\beta} .
\end{aligned}
$$

Corollary 8. Let $0<\inf p_{k}=h \leq p_{k} \leq \sup p_{k}=H<\infty$ and let $\mathscr{M}=\left(M_{k}\right)$ be Musielak-Orlicz function satisfying $\Delta_{2^{-}}$ condition. Then

$$
\begin{aligned}
& c_{0}^{\theta}\left[p, \Delta_{n}^{m}, q,\|\cdot, \ldots, \cdot\|\right]_{\alpha}^{\beta} \\
& \subset c_{0}^{\theta}\left[\mathscr{M}, p, \Delta_{n}^{m}, q,\|\cdot, \ldots, \cdot\|\right]_{\alpha}^{\beta}, \\
& c_{\infty}^{\theta}\left[p, \Delta_{n}^{m}, q,\|\cdot, \ldots, \cdot\|\right]_{\alpha}^{\beta} \\
& \quad \subset c_{\infty}^{\theta}\left[\mathscr{M}, p, \Delta_{n}^{m}, q,\|\cdot, \ldots, \cdot\|\right]_{\alpha}^{\beta} .
\end{aligned}
$$


Proof. The proof follows by taking $\mathscr{M}^{\prime}(x)=x$ in the above Theorem 7.

Theorem 9. If $\mathscr{M}=\left(M_{k}\right)$ be the Musielak-Orlicz function, then the following statements are equivalent:

(i) $c_{\infty}^{\theta}\left[p, \Delta_{n}^{m}, q,\|\cdot, \ldots, \cdot\|\right]_{\alpha}^{\beta} \subset c_{\infty}^{\theta}\left[\mathscr{M}, p, \Delta_{n}^{m}, q,\|\cdot, \ldots, \cdot\|\right]_{\alpha}^{\beta}$,

(ii) $c_{0}^{\theta}\left[p, \Delta_{n}^{m}, q,\|\cdot, \ldots, \cdot\|\right]_{\alpha}^{\beta} \subset c_{\infty}^{\theta}\left[\mathscr{M}, p, \Delta_{n}^{m}, q,\|\cdot, \ldots, \cdot\|\right]_{\alpha}^{\beta}$,

(iii) $\sup _{r}\left(1 / h_{r}^{\alpha}\right)\left[\sum_{k \in I_{r}}\left[M_{k}(t / \rho)\right]^{p_{k}}\right]^{\beta}<\infty(t, \rho>0)$.

Proof.

(i) $\Rightarrow$ (ii). It is obvious in view of the following fact that

$$
c_{0}^{\theta}\left[p, \Delta_{n}^{m}, q,\|\cdot, \ldots, \cdot\|\right]_{\alpha}^{\beta} \subset c_{\infty}^{\theta}\left[p, \Delta_{n}^{m}, q,\|\cdot, \ldots, \cdot\|\right]_{\alpha}^{\beta} .
$$

(ii) $\Rightarrow$ (iii). Let $c_{0}^{\theta}\left[p, \Delta_{n}^{m}, q,\|\cdot, \ldots, \cdot\|\right]_{\alpha}^{\beta} \subset c_{\infty}^{\theta}\left[\mathscr{M}, p, \Delta_{n}^{m}, q\right.$, $\|\cdot, \ldots, \cdot\|]_{\alpha}^{\beta}$. Assume that (iii) does not hold. Then, for some $t, \rho>0$, we obtain

$$
\sup _{r} \frac{1}{h_{r}^{\alpha}}\left[\sum_{k \in I_{r}}\left[M_{k}\left(\frac{t}{\rho}\right)\right]^{p_{k}}\right]^{\beta}=\infty
$$

and so one can find a subinterval $I_{r(j)}$ of the set of interval $I_{r}$ such that

$$
\frac{1}{h_{r(j)}^{\alpha}}\left[\sum_{k \in I_{r(j)}}\left[M_{k}\left(\frac{j^{-1}}{\rho}\right)\right]^{p_{k}}\right]^{\beta}>j, \quad j=1,2 .
$$

Let us define the sequence $x=\left(x_{k}\right)$ by

$$
\Delta_{n}^{m} x_{k+s}=\left\{\begin{array}{ll}
j^{-1}, & \text { if } k \in I_{r(j)}, \\
0, & \text { if } k \notin I_{r(j)},
\end{array} \quad \forall s \in \mathbb{N} .\right.
$$

Then $x=\left(x_{k}\right) \in c_{0}^{\theta}\left[p, \Delta_{n}^{m}, q,\|\cdot, \ldots, \cdot\|\right]_{\alpha}^{\beta}$ but, by (47), $x=$ $\left(x_{k}\right) \notin c_{\infty}^{\theta}\left[\mathscr{M}, p, \Delta_{n}^{m}, q,\|\cdot, \ldots, \cdot\|\right]_{\alpha}^{\beta}$ which contradicts (ii). Hence, the condition (iii) must hold.

(iii) $\Rightarrow$ (i). Suppose that (iii) holds and $x=\left(x_{k}\right) \in$ $c_{0}^{\theta}\left[p, \Delta_{n}^{m}, q,\|\cdot, \ldots, \cdot\|\right]_{\alpha}^{\beta}$. Also assume that $x=\left(x_{k}\right) \notin c_{\infty}^{\theta}[\mathscr{M}$, $\left.p, \Delta_{n}^{m}, q,\|\cdot, \ldots, \cdot\|\right]_{\alpha}^{\beta}$. Then

$$
\begin{aligned}
\sup _{r, s} & \frac{1}{h_{r}^{\alpha}}\left[\sum_{k \in I_{r}}\left[M_{k}\left(\left\|\frac{\Delta^{m} x_{k+s}}{\rho}, z_{1}, \ldots, z_{n-1}\right\|\right)\right]^{p_{k}}\right]^{\beta} \\
\quad=\infty . &
\end{aligned}
$$

Let $t=q_{k}\left(\left\|\Delta_{n}^{m} x_{k+s}, z_{1}, \ldots, z_{n-1}\right\|\right)$ for each $k$ and fixed $s$. It follows from (49) that

$$
\sup _{r} \frac{1}{h_{r}^{\alpha}}\left[\sum_{k \in I_{r}}\left[M_{k}\left(\frac{t}{\rho}\right)\right]\right]^{\beta}=\infty,
$$

which contradicts (iii). Hence, the condition (i) holds.
Theorem 10. Let $1 \leq p_{k} \leq \sup p_{k}<\infty$ and let $\mathscr{M}=\left(M_{k}\right)$ be a Musielak-Orlicz function. Then the following statements are equivalent:

(i) $c_{0}^{\theta}\left[\mathscr{M}, p, \Delta_{n}^{m}, q,\|\cdot, \ldots, \cdot\|\right]_{\alpha}^{\beta} \subset c_{0}^{\theta}\left[p, \Delta_{n}^{m}, q,\|\cdot, \ldots, \cdot\|\right]_{\alpha}^{\beta}$,

(ii) $c_{0}^{\theta}\left[\mathscr{M}, p, \Delta_{n}^{m}, q,\|\cdot, \ldots, \cdot\|\right]_{\alpha}^{\beta} \subset c_{\infty}^{\theta}\left[p, \Delta_{n}^{m}, q,\|\cdot, \ldots, \cdot\|\right]_{\alpha}^{\beta}$,

(iii) $\inf _{r}\left(1 / h_{r}^{\alpha}\right)\left[\sum_{k \in I_{r}}\left[M_{k}(t / \rho)\right]^{p_{k}}\right]^{\beta}>0(t, \rho>0)$.

Proof. It is trivial to obtain that (i) implies (ii). We are now proving that (ii) implies (iii). Let the condition (ii) hold. Assume that (iii) does not hold. Therefore,

$$
\inf _{r} \frac{1}{h_{r}^{\alpha}}\left[\sum_{k \in I_{r}}\left[M_{k}\left(\frac{t}{\rho}\right)\right]^{p_{k}}\right]^{\beta}=0 \quad(t, \rho>0)
$$

and so we can find a subinterval $I_{r(j)}(j=1,2)$ of $I_{r}$ such that

$$
\frac{1}{h_{r(j)}^{\alpha}}\left[\sum_{k \in I_{r(j)}}\left[M_{k}\left(\frac{j}{\rho}\right)\right]^{p_{k}}\right]^{\beta}<j^{-1} .
$$

For all $s \in \mathbb{N}$, define $\Delta_{n}^{m} x_{k+s}=j$ if $k \in I_{r(j)}$ and $\Delta_{n}^{m} x_{k+s}=$ 0 if $k \notin I_{r(j)}$. If follows from (52) that $x=\left(x_{k}\right) \in$ $c_{0}^{\theta}\left[p, \Delta_{n}^{m}, q,\|\cdot, \ldots, \cdot\|\right]_{\alpha}^{\beta}$ but $x=\left(x_{k}\right) \notin c_{\infty}^{\theta}\left[p, \Delta_{n}^{m}, q,\|\cdot, \ldots, \cdot\|\right]_{\alpha}^{\beta}$ which contradicts (ii). Thus, the condition (iii) holds. It is left to prove that (iii) implies (i). Suppose that (iii) holds and also assume that $x=\left(x_{k}\right) \in c_{0}^{\theta}\left[\mathscr{M}, p, \Delta_{n}^{m}, q,\|\cdot, \ldots, \cdot\|\right]_{\alpha}^{\beta}$. Therefore,

$$
\lim _{r \rightarrow \infty} \frac{1}{h_{r}^{\alpha}}\left[\sum_{k \in I_{r}}\left[M_{k}\left(q_{k}\left(\left\|\frac{\Delta_{n}^{m} x_{k+s}}{\rho}, z_{1}, \ldots, z_{n-1}\right\|\right)\right)\right]^{p_{k}}\right]^{\beta}
$$

$=0 \quad$ uniformly in $s$, for some $\rho>0$.

Again, assume that $x=\left(x_{k}\right) \notin c_{0}^{\theta}\left[p, \Delta_{n}^{m}, q,\|\cdot, \ldots, \cdot\|\right]_{\alpha}^{\beta}$. Then, for some number $\epsilon>0$ and a subinterval $I_{r(j)}$ of $I_{r}$, one obtains $\left\|\Delta_{n}^{m} x_{k+s}, z_{1}, \ldots, z_{n-1}\right\| \geq \epsilon$ for all $k \in \mathbb{N}$ and for some $s \geq s_{0}$. Therefore, in view of the properties of the Orlicz function, one writes

$$
\begin{aligned}
& {\left[M_{k}\left(q_{k}\left(\left\|\frac{\Delta_{n}^{m} x_{k+s}}{\rho}, z_{1}, \ldots, z_{n-1}\right\|\right)\right)^{p_{k}}\right]^{\beta}} \\
& \geq\left[M_{k}\left(\frac{\epsilon}{\rho}\right)^{p_{k}}\right]^{\beta} .
\end{aligned}
$$

By taking into account (53), we obtain

$$
\lim _{r \rightarrow \infty} \frac{1}{h_{r}^{\alpha}}\left[\sum_{k \in I_{r}}\left[M_{k}\left(\frac{\epsilon}{\rho}\right)\right]^{p_{k}}\right]^{\beta}=0
$$

which contradicts (iii). Hence, (i) must hold.

Theorem 11. Let $0<p_{k} \leq r_{k}$ for all $k \in \mathbb{N}$ and let $\left(r_{k} / p_{k}\right)$ be bounded. Then

$$
\begin{aligned}
c^{\theta} & {\left[\mathscr{M}, r, \Delta_{n}^{m}, q,\|\cdot, \ldots, \cdot\|\right]_{\alpha}^{\beta} } \\
& \quad c^{\theta}\left[\mathscr{M}, p, \Delta_{n}^{m}, q,\|\cdot, \ldots, \cdot\|\right]_{\alpha}^{\beta} .
\end{aligned}
$$


Proof. Suppose that $x \in c^{\theta}\left[\mathscr{M}, r, \Delta_{n}^{m}, q,\|\cdot, \ldots, \cdot\|\right]_{\alpha}^{\beta}$. Write

$$
\begin{aligned}
& t_{k} \\
& =\left[\left[M_{k}\left(q_{k}\left(\left\|\frac{\Delta_{n}^{m} x_{k+s}-L}{\rho}, z_{1}, \ldots, z_{n-1}\right\|\right)\right)\right]^{r_{k}}\right]^{\beta},
\end{aligned}
$$

and $\mu_{k}=p_{k} / r_{k}$ for all $k \in \mathbb{N}$. Then $0<\mu_{k} \leq 1$ for all $k \in \mathbb{N}$. Take $0<\mu<\mu_{k}$ for $k \in \mathbb{N}$. Consider two sequences $\left(u_{k}\right)$ and $\left(v_{k}\right)$ defined by $u_{k}=t_{k}$ and $v_{k}=0$ if $t_{k} \geq 1$ and $u_{k}=0$ and $v_{k}=t_{k}$ for $t_{k}<1$. Clearly

$$
\begin{gathered}
t_{k}=u_{k}+v_{k}, \\
t_{k}^{\mu_{k}}=u_{k}^{\mu_{k}}+v_{k}^{\mu_{k}},
\end{gathered}
$$

$\forall k \in \mathbb{N}$.

It follows that $u_{k}^{\mu_{k}} \leq u_{k} \leq t_{k}$ and $v_{k}^{\mu_{k}} \leq v_{k}^{\mu}$. Therefore,

$$
\frac{1}{h_{r}^{\alpha}} \sum_{k \in I_{r}} t_{k}^{\mu_{k}}=\frac{1}{h_{r}^{\alpha}} \sum_{k \in I_{r}}\left(u_{k}^{\mu_{k}}+v_{k}^{\mu_{k}}\right) \leq \frac{1}{h_{r}^{\alpha}} \sum_{k \in I_{r}} t_{k}+\frac{1}{h_{r}^{\alpha}} \sum_{k \in I_{r}} v_{k}^{\mu} .
$$

Now, for each $k$, we obtain

$$
\begin{aligned}
& \frac{1}{h_{r}^{\alpha}} \sum_{k \in I_{r}} v_{k}^{\mu}=\sum_{k \in I_{r}}\left(\frac{1}{h_{r}^{\alpha}} v_{k}\right)^{\mu}\left(\frac{1}{h_{r}^{\alpha}}\right)^{1-\mu} \\
& \quad \leq\left(\sum_{k \in I_{r}}\left[\left(\frac{1}{h_{r}^{\alpha}} v_{k}\right)^{\mu}\right]^{1 / \mu}\right)^{\mu} \\
& \quad \cdot\left(\sum_{k \in I_{r}}\left[\left(\frac{1}{h_{r}^{\alpha}}\right)^{1-\mu}\right]^{1 /(1-\mu)}\right)^{1-\mu}=\left(\frac{1}{h_{r}^{\alpha}} \sum_{k \in I_{r}} v_{k}\right)^{\mu} .
\end{aligned}
$$

By taking the above two inequalities into account, we get

$$
\frac{1}{h_{r}^{\alpha}} \sum_{k \in I_{r}} t_{k}^{\mu_{k}} \leq \frac{1}{h_{r}^{\alpha}} \sum_{k \in I_{r}} t_{k}+\left(\frac{1}{h_{r}^{\alpha}} \sum_{k \in I_{r}} v_{k}\right)^{\mu}
$$

Hence, we conclude that $x \in c^{\theta}\left[\mathscr{M}, p, \Delta_{n}^{m}, q,\|\cdot, \ldots, \cdot\|\right]_{\alpha}^{\beta}$.

\section{Theorem 12.}

(i) If $0<\inf p_{k} \leq p_{k} \leq 1$ for all $k \in \mathbb{N}$, then

$$
\begin{aligned}
c^{\theta} & {\left[\mathscr{M}, p, \Delta_{n}^{m}, q,\|\cdot, \ldots, \cdot\|\right]_{\alpha}^{\beta} } \\
& \subset c^{\theta}\left[\mathscr{M}, \Delta_{n}^{m}, q,\|\cdot, \ldots, \cdot\|\right]_{\alpha}^{\beta} .
\end{aligned}
$$

(ii) If $1 \leq p_{k} \leq \sup p_{k}<\infty$ for all $k \in \mathbb{N}$, then

$$
\begin{aligned}
& c^{\theta}\left[\mathscr{M}, \Delta_{n}^{m}, q,\|\cdot, \ldots, \cdot\|\right]_{\alpha}^{\beta} \\
& \quad \subset c^{\theta}\left[\mathscr{M}, p, \Delta_{n}^{m}, q,\|\cdot, \ldots, \cdot\|\right]_{\alpha}^{\beta} .
\end{aligned}
$$

Proof. (i) Assume that $x \in c^{\theta}\left[\mathscr{M}, p, \Delta_{n}^{m}, q,\|\cdot, \ldots, \cdot\|\right]_{\alpha}^{\beta}$. Then

$$
\begin{aligned}
& \lim _{r \rightarrow \infty} \frac{1}{h_{r}^{\alpha}}\left[\sum_{k \in I_{r}}\left[M_{k}\left(q_{k}\left(\left\|\frac{\Delta_{n}^{m} x_{k+s}-L}{\rho}, z_{1}, \ldots, z_{n-1}\right\|\right)\right)\right]^{p_{k}}\right]^{\beta} \\
& \quad=0 .
\end{aligned}
$$

Since $0<\inf p_{k} \leq p_{k} \leq 1$, we have

$$
\begin{aligned}
& \lim _{r \rightarrow \infty} \frac{1}{h_{r}^{\alpha}}\left[\sum_{k \in I_{r}}\left[M_{k}\left(q_{k}\left(\left\|\frac{\Delta_{n}^{m} x_{k+s}-L}{\rho}, z_{1}, \ldots, z_{n-1}\right\|\right)\right)\right]\right]^{\beta} \\
& \leq \lim _{r \rightarrow \infty} \\
& \cdot \frac{1}{h_{r}^{\alpha}}\left[\sum_{k \in I_{r}}\left[M_{k}\left(q_{k}\left(\left\|\frac{\Delta_{n}^{m} x_{k+s}-L}{\rho}, z_{1}, \ldots, z_{n-1}\right\|\right)\right)\right]^{p_{k}}\right]^{\beta} .
\end{aligned}
$$

Thus, we have

$$
\begin{aligned}
& \lim _{r \rightarrow \infty} \frac{1}{h_{r}^{\alpha}}\left[\sum_{k \in I_{r}}\left[M_{k}\left(q_{k}\left(\left\|\frac{\Delta_{n}^{m} x_{k+s}-L}{\rho}, z_{1}, \ldots, z_{n-1}\right\|\right)\right)\right]\right]^{\beta} \\
& \quad=0
\end{aligned}
$$

which gives $x \in c^{\theta}\left[\mathscr{M}, \Delta_{n}^{m}, q,\|\cdot, \ldots, \cdot\|\right]_{\alpha}^{\beta}$. Therefore, we conclude that

$$
\begin{aligned}
c^{\theta} & {\left[\mathscr{M}, p, \Delta_{n}^{m}, q,\|\cdot, \ldots, \cdot\|\right]_{\alpha}^{\beta} } \\
& \quad c^{\theta}\left[\mathscr{M}, \Delta_{n}^{m}, q,\|\cdot, \ldots, \cdot\|\right]_{\alpha}^{\beta} .
\end{aligned}
$$

(ii) Suppose that $p_{k} \geq 1$ for each $k$ and $\sup p_{k}<\infty$. Let $x \in c^{\theta}\left[\mathscr{M}, \Delta_{n}^{m}, q,\|\cdot, \ldots, \cdot\|\right]_{\alpha}^{\beta}$. Then, for each $\epsilon>0$ there exists a positive integer $N$ such that

$$
\lim _{r \rightarrow \infty} \frac{1}{h_{r}^{\alpha}}\left[\sum_{k \in I_{r}}\left[M_{k}\left(q_{k}\left(\left\|\frac{\Delta_{n}^{m} x_{k+s}-L}{\rho}, z_{1}, \ldots, z_{n-1}\right\|\right)\right)\right]^{p_{k}}\right]^{\beta}
$$


Since $1 \leq p_{k} \leq \sup p_{k}<\infty$, we have

$$
\begin{aligned}
\lim _{r \rightarrow \infty} \frac{1}{h_{r}^{\alpha}} & {\left[\sum_{k \in I_{r}}\left[M_{k}\left(q_{k}\left(\left\|\frac{\Delta_{n}^{m} x_{k+s}-L}{\rho}, z_{1}, \ldots, z_{n-1}\right\|\right)\right)\right]^{p_{k}}\right]^{\beta} } \\
& \leq \lim _{r \rightarrow \infty} \frac{1}{h_{r}^{\alpha}}\left[\sum_{k \in I_{r}}\left[M_{k}\left(q_{k}\left(\left\|\frac{\Delta^{m} x_{k+s}-L}{\rho}, z_{1}, \ldots, z_{n-1}\right\|\right)\right)\right]\right]^{\beta}=0<1 .
\end{aligned}
$$

Hence, $x \in c^{\theta}\left[\mathscr{M}, p, \Delta_{n}^{m}, q,\|\cdot, \ldots, \cdot\|\right]_{\alpha}^{\beta}$.

\section{Conflicts of Interest}

The authors declare that they have no conflicts of interest.

\section{Acknowledgments}

This project was funded by the Deanship of Scientific Research (DSR) at King Abdulaziz University, Jeddah, under Grant no. (RG-14-130-38). The authors, therefore, acknowledge with thanks DSR for technical and financial support.

\section{References}

[1] S. Gähler, "Lineare 2-normierte Räume," Mathematische Nachrichten, vol. 28, pp. 1-43, 1964.

[2] A. Misiak, " $n$-inner product spaces," Mathematische Nachrichten, vol. 140, pp. 299-319, 1989.

[3] H. Kızmaz, "On certain sequence spaces," Canadian Mathematical Bulletin, vol. 24, no. 2, pp. 169-176, 1981.

[4] M. Et and R. Çolak, "On some generalized difference sequence spaces," Soochow Journal of Mathematics, vol. 21, no. 4, pp. 377386, 1995.

[5] B. C. Tripathy and A. Esi, "A new type of difference sequence spaces," The International Journal of Science \& Technology, vol. 1, no. 1, pp. 11-14, 2006.

[6] B. C. Tripathy, A. Esi, and B. Tripathy, "On a new type of generalized difference Cesàro sequence spaces," Soochow Journal of Mathematics, vol. 31, no. 3, pp. 333-340, 2005.

[7] M. Et, "Generalized Cesàro difference sequence spaces of nonabsolute type involving lacunary sequences," Applied Mathematics and Computation, vol. 219, no. 17, pp. 9372-9376, 2013.

[8] S. A. Mohiuddine and B. Hazarika, "Some classes of ideal convergent sequences and generalized difference matrix operator," Univerzitet u Nišu. Prirodno-Matematički Fakultet. Filomat, vol. 31, no. 6, pp. 1827-1834, 2017.

[9] S. A. Mohiuddine and K. Raj, "Vector valued Orlicz-Lorentz sequence spaces and their operator ideals," Journal of Nonlinear Sciences and Applications. JNSA, vol. 10, no. 2, pp. 338-353, 2017.

[10] M. Mursaleen, S. K. Sharma, and A. Kiliçman, "Sequence spaces defined by musielak-orlicz function over n -normed spaces," Abstract and Applied Analysis, vol. 2013, Article ID 364743, 2013.

[11] M. Mursaleen and S. K. Sharma, "Entire sequence spaces defined on locally convex Hausdorff topological space," Iranian Journal of Science \& Technology, vol. 38, no. 2, pp. 105-109, 2014.

[12] M. A. Ragusa and A. Tachikawa, "On interior regularity of minimizers of $p(x)$-energy functionals," Nonlinear Analysis.
Theory, Methods \& Applications. An International Multidisciplinary Journal, vol. 93, pp. 162-167, 2013.

[13] K. Raj, A. K. Sharma, and S. K. Sharma, "A sequence space defined by Musielak-Orlicz function," International Journal of Pure and Applied Mathematics, vol. 67, no. 4, pp. 475-484, 2011.

[14] M. A. Krasnoselskii and J. B. Rutitsky, Convex functions and Orlicz spaces, P. Noordhoff Ltd., Groningen, Netherlands, 1961.

[15] F. Giannetti, A. Passarelli di Napoli, M. A. Ragusa, and A. Tachikawa, "Partial regularity for minimizers of a class of non autonomous functionals with nonstandard growth," Calculus of Variations and Partial Differential Equations, vol. 56, no. 6, Art. 153, 29 pages, 2017.

[16] J. Lindenstrauss and L. Tzafriri, "On Orlicz sequence spaces," Israel Journal of Mathematics, vol. 10, pp. 379-390, 1971.

[17] J. Musielak, Orlicz Spaces and Modular Spaces, vol. 1034 of Lecture Notes in Mathematics, Springer, Berlin, Germany, 1983.

[18] G. G. Lorentz, "A contribution to the theory of divergent sequences," Acta Mathematica, vol. 80, pp. 167-190, 1948.

[19] S. A. Mohiuddine, "An application of almost convergence in approximation theorems," Applied Mathematics Letters, vol. 24, no. 11, pp. 1856-1860, 2011.

[20] I. J. Maddox, "On strong almost convergence," Mathematical Proceedings of the Cambridge Philosophical Society, vol. 85, no. 2, pp. 345-350, 1979.

[21] A. Zygmund, "Trigonometrical Series, of Monografýas de Matemáticas," Warszawa-Lwow, vol. 5, 1935.

[22] H. Fast, "Sur la convergence statistique," Colloquium Mathematicum, vol. 2, pp. 241-244, 1951.

[23] H. Steinhaus, "Sur la convergence ordinaire et la convergence asymptotique," Colloquium Mathematicum, vol. 2, pp. 73-74, 1951.

[24] I. J. Schoenberg, "The integrability of certain functions and related summability methods," The American Mathematical Monthly, vol. 66, pp. 361-375, 1959.

[25] T. Šalát, "On statistically convergent sequences of real numbers," Mathematica Slovaca, vol. 30, no. 2, pp. 139-150, 1980.

[26] J. A. Fridy, "On statistical convergence," International Journal of Analysis and Applications, vol. 5, no. 4, pp. 301-313, 1985.

[27] U. Kadak and S. A. Mohiuddine, "Generalized statistically almost convergence based on the difference operator which includes the $(p, q)$-gamma function and related approximation theorems," Results in Mathematics, vol. 73, no. 9, 2018.

[28] T. Acar and S. A. Mohiuddine, "Statistical $(C, 1)(E, 1)$ summability and Korovkin's theorem," Filomat, vol. 30, no. 2, pp. 387-393, 2016.

[29] C. Belen and S. A. Mohiuddine, "Generalized weighted statistical convergence and application," Applied Mathematics and Computation, vol. 219, no. 18, pp. 9821-9826, 2013. 
[30] N. L. Braha, H. M. Srivastava, and S. A. Mohiuddine, "A Korovkin's type approximation theorem for periodic functions via the statistical summability of the generalized de la Vallée Poussin mean," Applied Mathematics and Computation, vol. 228, pp. 162-169, 2014.

[31] H. Çakalli, "Lacunary statistical convergence in topological groups," Indian Journal of Pure and Applied Mathematics, vol. 26, no. 2, pp. 113-119, 1995.

[32] R. Çolak and Ç. A. Bektas, " $\lambda$-statistical convergence of order $\alpha$," Acta Mathematica Scientia B, vol. 31, no. 3, pp. 953-959, 2011.

[33] O. H. Edely, S. A. Mohiuddine, and A. . Noman, "Korovkin type approximation theorems obtained through generalized statistical convergence," Applied Mathematics Letters, vol. 23, no. 11, pp. 1382-1387, 2010.

[34] M. Mursaleen and S. A. Mohiuddine, "On lacunary statistical convergence with respect to the intuitionistic fuzzy normed space," Journal of Computational and Applied Mathematics, vol. 233, no. 2, pp. 142-149, 2009.

[35] E. Savas and M. Et, "On $\left(\Delta_{\lambda}^{m}, I\right)$-statistical convergence of order $\alpha$," Periodica Mathematica Hungarica, vol. 71, no. 2, pp. 135-145, 2015.

[36] R. Çolak, Statistical convergence of order a, Modern Methods in Analysis and its Applications, Anamaya, New Delhi, India, 2010.

[37] H. Şenül, On statistical convergence of order $(\alpha, \beta)$, In review.

[38] A. R. Freedman, J. J. Sember, and M. Raphael, "Some Cesàrotype summability spaces," Proceedings of the London Mathematical Society. Third Series, vol. 37, no. 3, pp. 508-520, 1978.

[39] J. A. Fridy and C. Orhan, "Lacunary statistical convergence," Pacific Journal of Mathematics, vol. 160, no. 1, pp. 43-51, 1993.

[40] K. Raj, S. K. Sharma, and A. K. Sharma, "Difference sequence spaces in $n$-normed spaces defined by Musielak-Orlicz function," Armenian Journal of Mathematics, vol. 3, no. 3, pp. 127-141, 2010.

[41] K. Raj and S. K. Sharma, "Some seminormed difference sequence spaces defined by a Musielak-Orlicz function over $n$ normed spaces," Journal of Mathematics and Applications, vol. 38, pp. 115-131, 2015. 


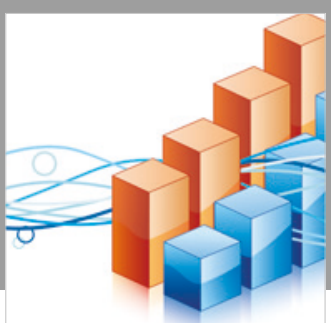

Advances in

Operations Research

\section{-n-m}
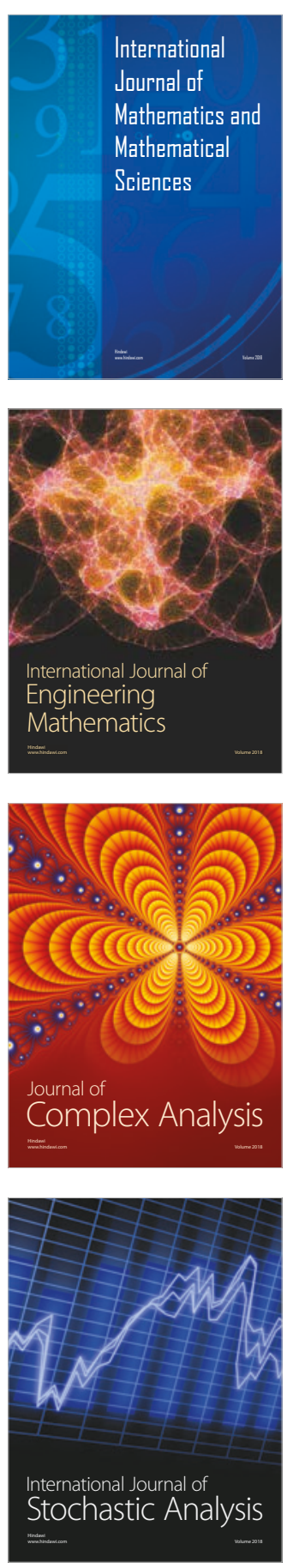
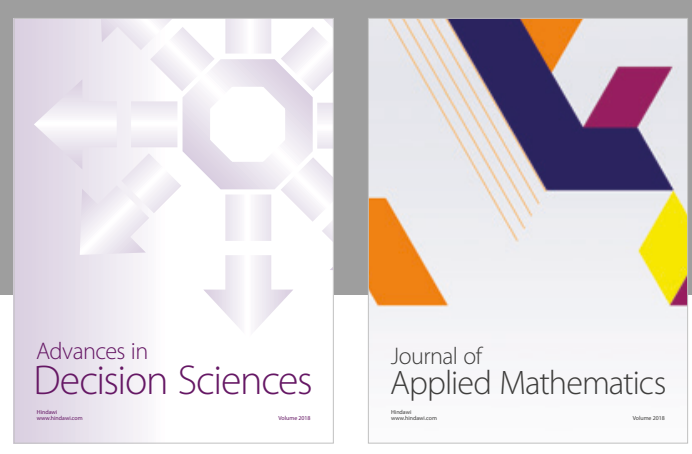

Journal of

Applied Mathematics
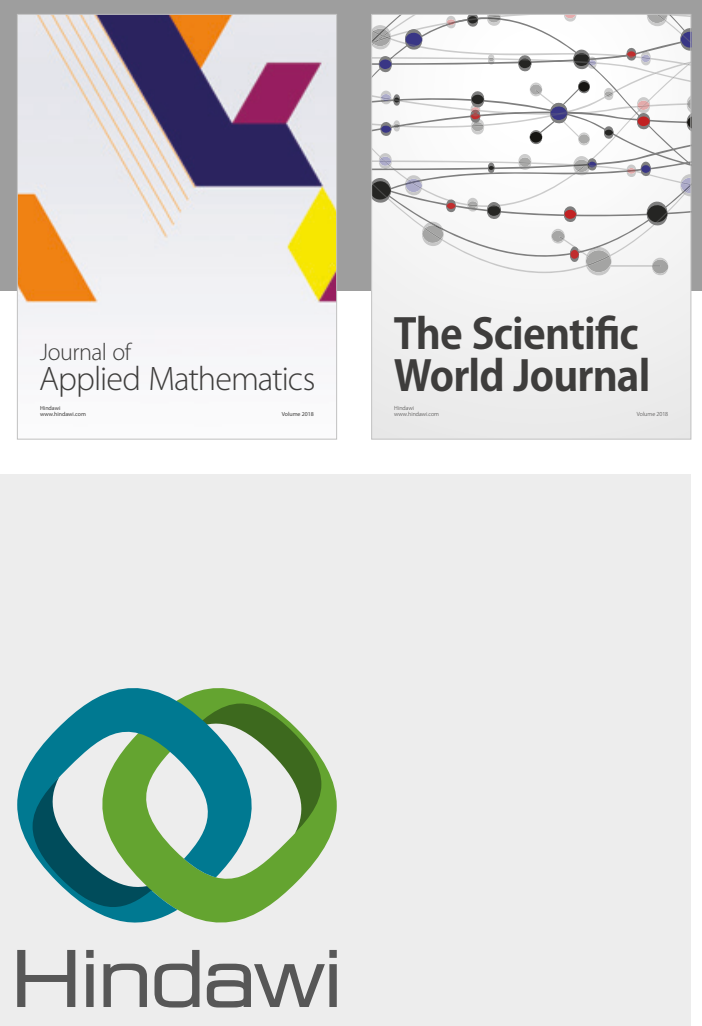

Submit your manuscripts at

www.hindawi.com

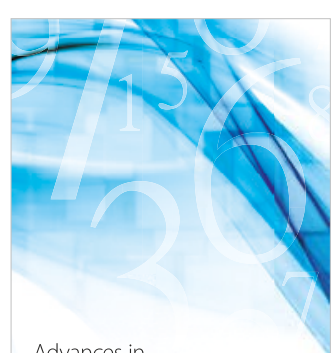

Advances in
Numerical Analysis
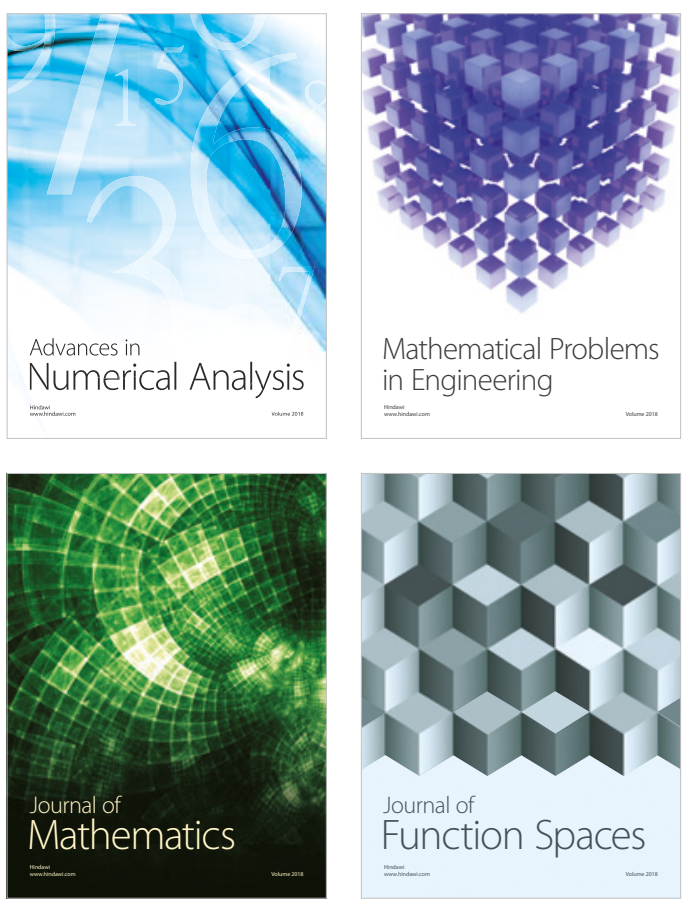

Mathematical Problems in Engineering

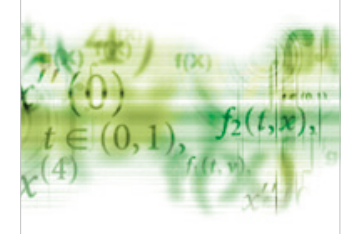

International Journal of

Differential Equations

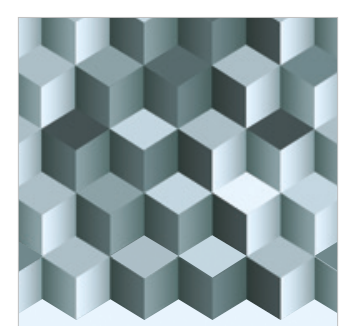

Journal of

Function Spaces

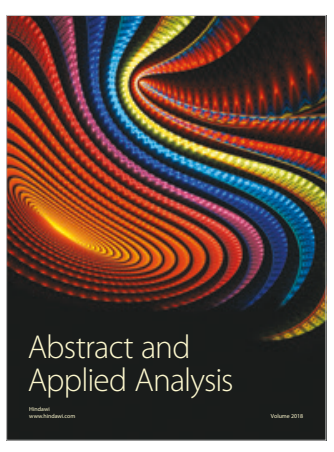

The Scientific

World Journal

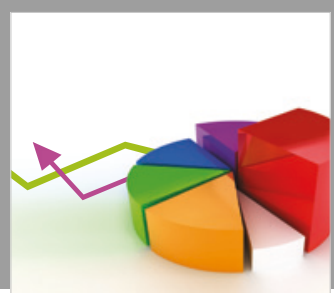

Journal of

Probability and Statistics
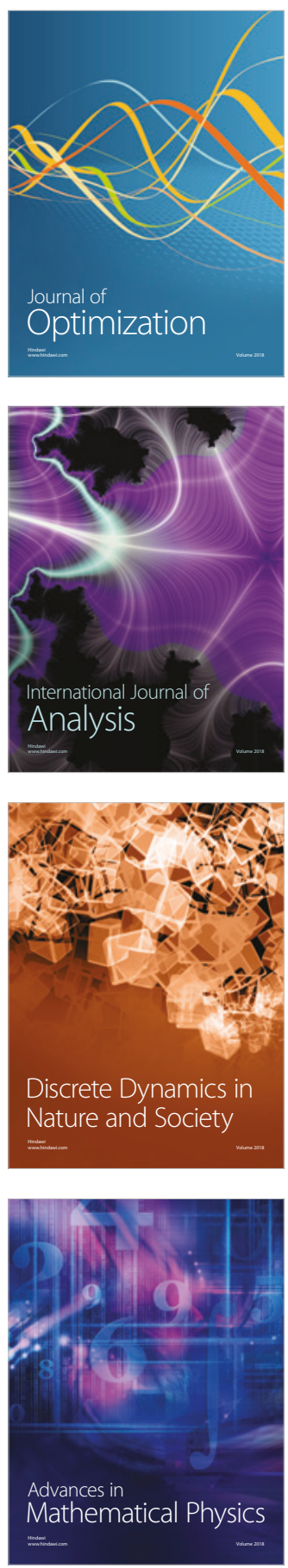